\title{
How to Solve a Multicriterion Problem for Which Pareto Dominance Relationship Cannot Be Applied? A Case Study from Medicine
}

\author{
Crina Grosan ${ }^{1}$, Ajith Abraham ${ }^{2}$, Stefan Tigan ${ }^{3}$, and Tae-Gyu Chang ${ }^{1}$ \\ ${ }^{1}$ Department of Computer Science \\ Babeş-Bolyai University, Cluj-Napoca, 3400, Romania \\ ${ }^{2}$ IITA Professorship Program, School of Computer Science and Engineering \\ Chung-Ang University, Seoul 156-756, Korea \\ ${ }^{3}$ Department of Medicine \\ University Iuliu Hatieganu, Cluj-Napoca, 3400, Romania \\ cgrosan@cs.ubbcluj.ro
}

\begin{abstract}
The most common way to deal with a multiobjective optimization problem is to apply Pareto dominance relationship between solutions. The question is: how can we make a decision for a multiobjective problem if we cannot use the conventional Pareto dominance for ranking solutions? We will exemplify this by considering a multicriterion problem for a medical domain problem. Trigeminal Neuralgia (TN) is a pain that is described as among the most acute known to mankind. TN produces excruciating, lightning strikes of facial pain, typically near the nose, lips, eyes or ears. Essential trigeminal neuralgia has questioned treatment methods. We consider five different treatment methods of the essential trigeminal neuralgia for evaluation under several criteria. We give a multiple criteria procedure using evolutionary algorithms for ranking the treatment methods of the essential trigeminal neuralgia for the set of all evaluation criteria. Results obtained by our approach using a very simple method are the same as the results obtained by applying weighted sum method (which requires lots of domain expert input). The advantage of the new proposed technique is that it does not require any additional information about the problem (like weights for each criteria in the case of weighted sum approach).
\end{abstract}

\section{Introduction}

It is generally accepted that multicriterion optimization in its present sense originated towards the end of the last century when Pareto (1848-1923) presented a qualitative definition for the optimality concept in economic problems with several competing criteria.

Instead of one scalar objective function, usually several conflicting and often non-commensurable (i.e. such quantities which have different units) criteria appear in an optimization problem. This situation forces the designer to look for a good compromise solution by considering tradeoffs between the competing criteria. Consequently, he must take a decision-maker's role in an interactive design 
process where typically several optimization problems must be solved. Multicriterion (multiobjective, Pareto, vector) optimization offers a flexible approach for the designer to treat such an overall decision-making problem in a systematic way $[9,[10$.

There are some particular situations for which Pareto dominance cannot be applied. This paper analyzes a multiobjective optimization problem for a medical domain problem. As evident from the considered test data, Pareto dominance cannot be applied in its initial form for classifying these treatments. The result will be that all solutions are non-dominated (which, in fact, means that all treatments are equal). This paper proposes an evolutionary algorithm to rank these treatments. A new dominance concept between two solutions is used. Results obtained are similar to the ones obtained by applying the weighted sum method.

The paper is structured as follows. Section 2 provides a short description of the Trigeminal Neuralgia. Sections 3 and 4 explain the scope of the present research and the motivation of the work done. Section 5 introduces and explains our approach. Section 6 presents the weighted sum method used for comparing the results of the proposed approach. Section 7 is dedicated to the experiments. Section 8 contains discussions and conclusions of the paper.

\section{What Is Trigeminal Neuralgia?}

Trigeminal neuralgia, also called tic douloureux, is a condition that affects the trigeminal nerve (the 5th cranial nerve), one of the largest nerves in the head. The trigeminal nerve is responsible for sending impulses of touch, pain, pressure, and temperature to the brain from the face, jaw, gums, forehead, and around the eyes. Trigeminal neuralgia is characterized by a sudden, severe, electric shocklike or stabbing pain typically felt on one side of the jaw or cheek. The disorder is more common in women than in men and rarely affects anyone younger than 50. The pain produced by trigeminal neuralgia is excruciating, perhaps the worst pain know to human beings. The attacks of pain, which generally last several seconds and may be repeated one after the other, may be triggered by talking, brushing teeth, touching the face, chewing, or swallowing. The attacks may come and go throughout the day and last for days, weeks, or months at a time, and then disappear for months or years [1, [5, 7]. Treatment for trigeminal neuralgia typically includes anticonvulsant medications such as carbamazepine or phenytoin. Baclofen, clonazepam, gabapentin, and valproic acid may also be effective and may be used in combination to achieve pain relief.

\section{Scope of Our Research}

The problem studied is the treatment of essential trigeminal neuralgia. For the treatment of essential trigeminal neuralgia many methods can be applied. The chronic evolution of the disease, its idiopathic character and the variable response to different treatment methods creates many disputes in the scientific world. The 
evaluation of the treatment methods from multiple points of view is difficult and has a high degree of subjectivity. The complex and original study with many patients, over the usual number from related studies, can contribute greatly to the evolution of this domain. The problem is to rank these treatments subject to multiple criteria.

\section{Motivation of the Work}

The problem of effectively ranking several treatments for Trigeminal Neuralgia could be formulated as a multiobjective optimization problem due to the number of different criteria which have to be satisfied simultaneously. The case analyzed in this research is a real problem [2. The most common approaches of a multiobjective optimization problem use the concept of Pareto dominance as defined below:

Definition (Pareto dominance).

Consider a maximization problem. Let $x, y$ be two decision vectors (solutions) from the definition domain. Solution $x$ dominate $y$ (also written as $x \succ y$ ) if and only if the following conditions are fulfilled:

(i) $f_{i}(x) \geq f_{i}(y), \forall i=1,2, \ldots, n$,

(ii) $\exists j \in\{1,2, \ldots, n\}: f_{j}(x)>f_{j}(y)$.

$n$ denotes the number of objectives. That is, a feasible vector $x$ is Pareto optimal if no feasible vector $y$ can increase some criterion without causing a simultaneous decrease in at least one other criterion.

As evident from the experiment section (Table 1), if we are applying the classical Pareto definition in order to obtain a hierarchy of treatments, all solutions will appear as non-dominated. This way, it is difficult to say one solution is better than the other. Consequently, any of the existing algorithms dealing with multiobjective problems from a Pareto dominance perspective cannot be applied. One solution is to use some of the traditional mathematical approaches which combine objectives and reduce the problem to a single objective optimization problem. But in this situation, additional information about the problem is required. For instance, every common approach will need details about the importance of each of the criteria. In this situation, a weight (i.e. a real number between 0 and 1 ) will be assigned to each criterion. This weight represent the importance (or the percentage) of that criteria between all criteria considered (sum of all these weights is equal to 1 ).

But in the case analyzed in this paper, all criteria are important since all of them are direct consequences of a treatment applied. So, finding a weight for each criterion is an extra task and can be sometimes difficult to assign.

\section{Proposed Approach}

An Evolutionary Algorithm (EA) 3], 4] approach is proposed for solving this problem. The population is initially randomly generated over the search space 
which is the definition domain. By applying genetic operators (like selection, mutation, crossover, etc.) these solutions (called also chromosome, individuals) are improved. Each individual from population is evaluated by using a quality (fitness) function. Using this quality the best individuals are selected at each generation.

Since, in our case, the final solution has to be a hierarchy of the treatments used, a chromosome (or a solution) will consist of a string representing a permutation of these treatments. For each pair of consecutive genes we will compute the number of objectives for which one is better than the other. We finally want to obtain a decreasing order of the treatments efficiency. The fitness (quality) of a chromosome will be equal to the number of treatments which are not arranged in a decreasing order (as compared with the successor). The quality zero certifies that the treatments are decreasingly arranged while taking into account the efficiency.

The algorithm works as follows: The initial population is generated. The only genetic operator used is mutation which consists of exchanging values of two genes randomly generated. Each individual is affected by mutation with a given probability. Parent and offspring are compared using the dominance concept presented above. The best between parent and offspring will be kept in the population of the next generation. This process is repeated for a given number of generations.

\section{Weighted Sum Approach}

The weighted-sum method is a traditional, popular method that parametrically changes the weights among objective functions to obtain the Pareto front [6]. Let us consider we have the objective functions $f_{1}, f_{2}, \ldots, f_{n}$. This method takes each objective function and multiplies it by a fraction of one, the "weighting coefficient" which is represented by $w_{i}$. The modified functions are then added together to obtain a single cost function, which can easily be solved using any method which can be applied for single objective optimization.

Mathematically, the new function is written as:

$$
\begin{aligned}
& \sum_{i=1}^{n} w_{i} f_{i}, \\
& \text { where } 0 \leq w_{i} \leq 1 \\
& \text { and } \sum_{i=1}^{n} w_{i}=1 .
\end{aligned}
$$

The initial work using the weighted sum method was done by Zadeh [11. The method is simple to understand and easy to implement. The weight itself reflects the relative importance (preference) among the objective functions under consideration. However, there are several disadvantages of this technique:

- The user always has to specify the weights values for functions and sometimes this will not have any relationship with the importance of the objectives; 
- Non-convex parts of the Pareto set cannot be obtained by minimizing convex combinations of the objectives;

- A single solution is obtained at one time. If we are interested in obtaining a set of feasible solutions, the algorithm has to be run several times. This also, is not a warranty that the solutions obtained in different runs are different.

\section{Case Study}

We made a clinical study of the following treatment methods of essential trigeminal neuralgia: infiltrations with streptomycin, low level laser therapy, treatment by skin graft, treatment by sciatic nerve graft, treatment by neurectomy [2]. Among these treatments, neurectomy was considered a mutilating treatment and the other methods were considered conservative. The research was done on a number of 251 patients suffering from essential trigeminal neuralgia and took over 8 years. The data used in experiment represents real data and are adapted from [2]. In order to mark out the effects and results of these treatments seven evaluation criteria were considered: hospitalization period, remission period, pain relief, decrease in the number of crises, decrease in pain level, decrease of the pain area, decrease in medication. The application of the treatments was based on regular techniques and personal contributions. The evaluation matrix case is presented in Table 1

Table 1. Data considered

\begin{tabular}{|c|c|c|c|c|c|c|}
\hline CRITERIA & \multirow[t]{3}{*}{$\begin{array}{l}\text { Criterion } \\
\text { type }\end{array}$} & \multicolumn{4}{|c|}{ TREATMENT } & \multirow[b]{2}{*}{$\begin{array}{l}\text { itTreatment } \\
\text { by } \\
\text { neurec- } \\
\text { tomy }\end{array}$} \\
\hline & & $\begin{array}{l}\text { Infiltra- } \\
\text { tions } \\
\text { with } \\
\text { strepto- } \\
\text { mycin }\end{array}$ & $\begin{array}{l}\text { Low level } \\
\text { laser } \\
\text { therapy }\end{array}$ & $\begin{array}{l}\text { Treatmer } \\
\text { by skir } \\
\text { graft }\end{array}$ & $\begin{array}{l}\text { tTreatment } \\
\text { by sciatic } \\
\text { nerve } \\
\text { graft }\end{array}$ & \\
\hline & & $T_{1}$ & $\boldsymbol{T}_{2}$ & $T_{3}$ & $T_{4}$ & $T_{5}$ \\
\hline $\begin{array}{l}\text { Hospitalization } \\
\text { period } \quad C_{1}\end{array}$ & $\overline{m i n}$ & 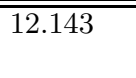 & 13.625 & 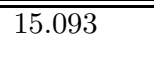 & 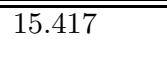 & 16.778 \\
\hline $\begin{array}{l}\text { Remission } \\
\text { period } C_{2}\end{array}$ & $\min$ & 9.964 & 10.453 & 12.07 & 11.889 & 12.022 \\
\hline $\begin{array}{l}\text { Pain } \\
\text { lief }\end{array} \stackrel{C_{3}}{ }$ & $\max$ & 21 & 18.34 & 25.2 & 34.742 & 18.457 \\
\hline $\begin{array}{l}\text { Number } \\
\text { of crises }\end{array}$ & $\max$ & 6.667 & 6.688 & 11.209 & 9.75 & 7.244 \\
\hline $\begin{array}{l}\text { Pain level } \\
\qquad C_{5}\end{array}$ & $\max$ & 3.423 & 3.281 & 3.558 & 3.833 & 3.156 \\
\hline $\begin{array}{l}\text { Pain area } \\
\qquad \boldsymbol{C}_{6}\end{array}$ & $\max$ & 0.904 & 0.937 & 0.937 & 0.978 & 0.848 \\
\hline $\begin{array}{r}\text { Medication } \\
\qquad C_{7}\end{array}$ & $\max$ & 364.286 & 442.188 & 655.814 & 586.111 & 255.556 \\
\hline
\end{tabular}




\subsection{Weighted Sum Approach Evaluation}

As evident from Table1, first two objectives have to be minimized and last 5 objectives have to be maximized. Weighted sum approach considers all objectives as having the same optimization criterion (minimization or maximization). For this purpose, we consider $-f_{1}$ and $-f_{2}$ instead of $f_{1}$ and $f_{2}$. This way, all objectives have to be maximized. In order to apply the weighted sum method, we need to specify a weight for each criterion. For the seven studied criteria we established specific values in the interval 0.02 and 0.54 . For the hospitalization period and for the remission period the values were more relevant as they decreased. For the other evaluated parameters, higher values expressed a good efficiency of the evaluated treatment method. Table 2 contains the values of the weights for each objective.

Table 2. Values of weights

\begin{tabular}{lccccccc}
\hline & & \multicolumn{7}{c}{ Criterion } \\
& $C_{1}$ & $C_{1}$ & $C_{1}$ & $C_{1}$ & $C_{1}$ & $C_{1}$ & $C_{1}$ \\
\hline \hline Weight & 0.08 & 0.06 & 0.54 & 0.08 & 0.17 & 0.05 & 0.02 \\
\hline
\end{tabular}

Results obtained by applying weighted sum approach are presented in Table 3 .

Table 3. Results obtained by applying weighted sum approach

\begin{tabular}{lllllllll}
\hline Treatment & \multicolumn{9}{c}{ Criterion } & & \multicolumn{2}{c}{$\begin{array}{c}\text { Weighted } \\
\text { sum }\end{array}$} \\
& $C_{1}$ & $C_{2}$ & $C_{3}$ & $C_{4}$ & $C_{5}$ & $C_{6}$ & $C_{7}$ & \\
\hline \hline$T_{1}$ & 12.143 & 9.964 & 21.0 & 6.667 & 3.423 & 0.904 & 364.286 & $\mathbf{1 8 . 2 1 6 9 1}$ \\
$T_{2}$ & 13.635 & 10.453 & 18.34 & 6.688 & 3.281 & 0.922 & 442.188 & $\mathbf{1 8 . 1 6 8 2 9}$ \\
$T_{3}$ & 15.093 & 12.07 & 25.2 & 11.209 & 3.558 & 0.937 & 655.814 & $\mathbf{2 6 . 3 4 1 0 7}$ \\
$T_{4}$ & 15.417 & 11.889 & 34.742 & 9.75 & 3.833 & 0.978 & 586.111 & $\mathbf{3 0 . 0 1 6 7 1}$ \\
$T_{5}$ & 16.778 & 12.022 & 18.457 & 7.244 & 3.156 & 0.848 & 255.556 & $\mathbf{1 4 . 1 7 2 7 8}$ \\
\hline Weights & 0.08 & 0.06 & 0.54 & 0.08 & 0.17 & 0.05 & 0.02 & \\
\hline
\end{tabular}

As evident from Table 3, the ranking of the above treatments in decreasing order of its efficiency obtained by applying weighted sum approach is: $T_{4}$ (Sciatic nerve graft), $T_{3}$ (Skin graft), $T_{1}$ (Streptomycin), $T_{2}$ (Laser) and $T_{5}$ (Neurectomy).

\subsection{Proposed Approach Evaluation}

A sample solution obtained by the evolutionary algorithm used for the treatments ranking is:

$$
\left(T_{5}, T_{4}, T_{2}, T_{1}, T_{3}\right)
$$

Base on the relationships between solutions as presented in Table 4 , the fitness of this sample solution which is given by the number of treatments (genes) which are not in a decreasing order is equal to 3 . 
Table 4. Number of objectives (criteria) in which treatment $T_{i}$ dominates treatment $T_{j}$ (out of seven)

\begin{tabular}{llllll}
\hline Treatment & $\boldsymbol{T}_{1}$ & $\boldsymbol{T}_{2}$ & $\boldsymbol{T}_{3}$ & $\boldsymbol{T}_{4}$ & $\boldsymbol{T}_{5}$ \\
\hline \hline $\boldsymbol{T}_{1}$ & - & 4 & 2 & 2 & 6 \\
$\boldsymbol{T}_{2}$ & 3 & - & 2 & 2 & 5 \\
$\boldsymbol{T}_{3}$ & 5 & 5 & - & 3 & 7 \\
$\boldsymbol{T}_{4}$ & 5 & 5 & 4 & - & 7 \\
$\boldsymbol{T}_{5}$ & 1 & 2 & 0 & 0 & - \\
\hline
\end{tabular}

The parameters used by the evolutionary algorithm are:

- Population size: 20

- Number of generations: 20

- Mutation probability: 0.5.

- Crossover probability: 0.7 .

The hierarchy of treatments efficiency obtained is: $T_{4}, T_{3}, T_{1}, T_{2}, T_{5}$.

As evident from these experiments, both algorithms obtained same hierarchy for the treatments. The evolutionary algorithm is very simple and works very well for the considered data. Evolutionary algorithms can detect several solutions in a single run and the user could select the desired solution based on the problem constraints, feasibility and other criteria. But weighted sum approach (and other approaches for multiobjective optimization which are not using evolutionary computation) has to be applied several times in order to obtain multiple solutions. The common procedure involves exchanging objective's weights. But this can be problematic for the case studied due to the importance of objectives. For instance, we considered decreasing of pain criteria as being a very important one (weight is 0.54) and medication period (or hospitalization period) as having less importance (weights are 0.08 and 0.02 respectively). If we will exchange pain weight and medication weight we cannot have any warranty of the result quality.

\section{Discussions and Conclusions}

The patients had different responses to the same treatment method during the treatment period. This observation can be also found in other studies such as 8. The inadequate dosing of medications or treatments can also lead to failure [12. By applying evolutionary algorithms, the ranking of treatments efficiency obtained is similar to the one obtained by applying a standard mathematical approach for multiobjective optimization, namely the weighted sum approach. But the advantage is that we do not require any additional information about the problem while weighted sum approach involves a weight for each objective. This task can be sometimes difficult to achieve due to the objectives importance.

By combining all objectives in a single objective function (and transforming the multiobjective optimization problem into a single objective one) at most one solution could be obtained by the execution of the algorithm. In order to obtain 
multiple solutions, we have to apply the algorithm several times. Even then, we cannot be sure that all solutions are different. Running time required is another disadvantage of the weighted sum approach.

\section{Acknowledgements}

This research was supported by the International Joint Research Grant of the Institute of Information Technology Assessment foreign professor invitation program of the Ministry of Information and Communication, Korea. Authors would also like to thank Radu Campian, Grigore Baciut and Mihaela Baciut of the Department of Maxillofacial Surgery, University of Medicine and Pharmacy, Iuliu Hatieganu Cluj-Napoca, for the initial contributions of this research [2].

\section{References}

1. Apfelbaum, R.I. Trigeminal Neuralgia : Vascular Decompression. Carter and Spetzler - Neurovascular Surgery. Mc Graw Hill. International edition, pp. 1107-18, 1995.

2. Campian, R., Baciut, G., Baciut, M., Tigan, S. Pain evaluation in essential trigeminal neuralgia of essential trigeminal neuralgia treatments, Applied Medical Informatics, 15(3-4) pp. 21-25, 2004.

3. Goldberg, D.E. Genetic algorithms in search, optimization and machine learning. Addison Wesley, Reading, MA, 1989.

4. Holland, J. Adaptation in Natural and Artificial Systems, University of Michigan Press, Ann Arbor, 1975.

5. Jannetta, P.J. Microvascular Decompression of The Trigeminal Nerve for Tic Douloreux. Youmans - Neurological Surgery. Saunders Company, Fourth edition, 5, pp $3404-3415,1996$.

6. Kim, I.Y., de Weck, O.L. Adaptive weighted-sum method for bi-objective optimization: Pareto front generation. Structural and Multidisciplinary Optimization, 29, pp. 149-158, 2005.

7. Kondo, A. Follow-up Results in Microvascular Decompression in Trigeminal Neuralgia and Hemifacial Spasm. Neurosurgery, vol. 40; pp. 46 - 52, 1997.

8. Lee K.H. Facial pain: trigeminal neuralgia, Annals of the Academy of Medicine, Singapore, 22(2): 193-196, 1993.

9. Stadler, W. A Survey of Multicriteria Optimization, or the Vector Maximum Problem. JOTA 29, 1-52, 1979.

10. Steuer, R.E. Multiple Criteria Optimization: Theory, Computation and Application. New York: Wiley, 1986.

11. Zadeh, L. Optimality and Non-Scalar-Valued Performance Criteria. IEEE Transaction on Automation Control 8, 59-60, 1963.

12. Zakrzewska J.M., Patsalos P.N., Drugs used in the management of the trigeminal neuralgia, Oral Surgery, Oral Medicine, Oral Pathology, 74(4): 439-450, 1992. 